\title{
Specific and Non-Specific Factors of Humoral Immunity as Markers for Pregnancy Loss in Women with Cytomegalovirus Infection
}

\author{
Irina A. Andrievskaya, $\mathrm{PhD}, \mathrm{ScD}$; Michael T. Lucenko, $\mathrm{PhD}, \mathrm{ScD}$; \\ Olga P. Babenko, PhD \\ Far Eastern Scientific Center of Physiology and Pathology of Respiration, \\ Siberian Branch of Russian Academy of Sciences \\ Blagoveshchensk, the Russian Federation
}

\begin{abstract}
The aim of this study was to estimate the changes in humoral immunity and their association with complications of pregnancy (spontaneous abortions, threatened miscarriage, preterm birth) depending on the gestational age and recurrence of cytomegalovirus infection (CMVI).

A direct relationship between the frequency of detection of an anti-CMV IgG antibody titer of 1:1600 and the prevalence of acute respiratory disease during pregnancy has been identified. We found an imbalance in the production of the non-specific antibodies (an increase in the blood levels of total $\operatorname{IgM}$ and a decrease in $\operatorname{IgA}$ and $\operatorname{IgG}$ levels) in the subgroup of women with relapsed CMVI at 6 to 8 weeks of gestation and spontaneous abortion, as well as in the subgroup of women with relapsed CMVI at 15 to 21 weeks of gestation and the risk of the late miscarriage, compared to those with relapsed CMVI at 9 to 14 weeks and 22 to 32 weeks of gestation. An increase in blood levels of total IgM and IgG and a decrease in IgA level was identified in the subgroup of women with relapsed CMVI at 9 to14 weeks of gestation and a threatened abortion, as well as in the subgroup of women with relapsed CMVI at 22 to 32 weeks of gestation and preterm birth. The obtained data of the imbalance in the primary and secondary immune response in CMV-seropositive pregnant women during relapsed CMVI indicate disturbances in the systemic and local intercellular interactions of immunocompetent cells, which lead to an imbalance in the production of antibodies involved in the elimination of viral agents and to the development of a systemic inflammatory response that complicates the course of pregnancy. CMVI relapse at 7 to 8 weeks of gestation is associated with reproductive losses; a risk for threatened miscarriage, threatened premature labor, and retrochorial hematoma increases significantly with CMVI relapse in the more remote gestational age. (Int J Biomed. 2015;5(4):184-187.)
\end{abstract}

Keywords: cytomegalovirus infection; humoral immunity; spontaneous abortions; threatened miscarriage; preterm birth.

\section{Introduction}

Cytomegalovirus (CMV) is an extremely common virus that can infect almost anyone; about $60 \%-80 \%$ of women of childbearing age have antibodies to CMV [1,2]. According to the International Classification, CMV belongs to the type 5 of Human Herpesviruses (HHV) - CMV/HHV-5. CMV has the ability to remain latent within the body over long periods. CMV activation is associated with immunosuppression and hormonal changes [1].

*Corresponding author: Prof. Michael T. Lucenko, PhD, ScD, Academician of RAS, Head of Far Eastern Scientific Center of Physiology and Pathology of Respiration SB of RAS, Blagoveshchensk, Russia.E-mail:_Lucencomt@mail.ru
Among the factors of physiological immunosuppression which contribute to the spread of cytomegalovirus infection (CMVI) and its reactivation, the first place belongs to pregnancy $[3,4]$. This circumstance predetermines a special interest in the problem of CMVI during pregnancy $[5,6]$. Physiological immunodeficiency that occurs during pregnancy creates an increased risk of manifestation and recurrence of CMVI in pregnant women. The virus causes significant disturbances in the regulation of immune response, which are based on its property to cause immunosuppression $[7,8]$. Intracellular persistence of the virus contributes to the long latency of infection $[9,10]$. The intracellular location protects the virus from the action of specific antibodies. Changes in humoral immunity and their association with pregnancy, depending on the gestational age and recurrence of CMVI, have not been 
fully disclosed. In particular, there is no consensus regarding the high titer antibodies to CMV in pregnant women. Some authors believe that a high antibody titer has an adverse prognostic significance for the fetus, while other researchers believe that these antibodies penetrate the placenta and protect the fetus from infection $[7,11]$.

The aim of this study was to estimate the changes in humoral immunity and their association with complications of pregnancy (spontaneous abortions, threatened miscarriage, premature birth) depending on the gestational age and recurrence of CMVI.

\section{Materials and Methods}

This prospective study (between 2011 and 2013) included $165 \mathrm{CMV}$-seropositive pregnant women (the study group) and $50 \mathrm{CMV}$-seronegative pregnant women (the control group). The mean age of pregnant women in the study group was $24.6 \pm 0.4$ years and $23.5 \pm 0.5$ years in the control group $(P>0.05)$.

Inclusion criteria for the study group were a relapse of CMVI identified by molecular biological and serological methods, as well as herpes virus infection (HHV-1,2) remission during the entire gestation period.

Exclusion criteria were primary CMVI, an aggravation of other inflammatory diseases of extragenital localization and sexually transmitted infections, genetic/endocrinological reasons for complications of pregnancy, cervical incompetence, and the presence of uterine pathology (malformations, hypoplasia of uterus, intrauterine adhesions).

Blood samples $(5 \mathrm{ml})$ were collected from the ulnar vein in standard vacuum tubes with EDTA to obtain the samples of mononuclear cells. For serological tests, we used blood that does not contain anticoagulants. Mononuclear cell isolation for PCR was carried out with Ficoll-Urografin $(\mathrm{d}-1,077 \mathrm{~g} / \mathrm{ml})$ ("DNA-Technology", Moscow). The verification of CMV, the definition of type-specific antibody, the total immunoglobulin ( $\operatorname{Ig} \mathrm{A}, \mathrm{IgG}, \mathrm{IgM}$ ), and the avidity index were determined by ELISA on the spectrophotometer "Stat Fax 2100" (USA) using the sets of CC "Vector-Best" (Novosibirsk, Russia). CMV DNA was detected by PCR on the machine DT-96 using sets of "DNA-Technology" (Moscow, Russia). The relapse of CMVI was diagnosed in a comprehensive study of the peripheral blood to check for the presence of IgM or a fourfold or more increase in the $\mathrm{IgG}$ antibody titer in paired serum in the dynamics after 10 days, an avidity index $>65 \%$, and the presence of CMV DNA in samples of blood, urine, buccal epithelium, and cervical mucosa.

The clinical relapse of CMVI was manifested by acute respiratory disease. HHV infection type 1 and 2 in the stage of stable remission (anti-HHV-1,2 IgG antibody titer of 1:800, an avidity index $>65 \%$ ) was found in all patients at the first examination.

In the study group, in the first phase, the patients were divided into subgroups according to the course of pregnancy. Subgroup 1 included patients with reproductive losses $(n=15)$; Subgroup 2 included patients without reproductive losses $(\mathrm{n}=150)$. At the second stage, we formed the sub-subgroups within subgroups, taking into account the timing of CMVI relapse during gestation. In particular, the relapse of CMVI occurred at 7 and 8 weeks of gestation among all patients of Subgroup 1. Subgroup 2 was divided into the following subsubgroups: Subgroup 2a $(\mathrm{n}=50)$ with CMVI relapse at 9 and 14 weeks of gestation, Subgroup $2 b(n=50)$ at 15 and 21 weeks of gestation, and Subgroup $2 \mathrm{c}(\mathrm{n}=50)$ at 22 and 32 weeks of gestation.

The study was conducted in the laboratory of pathogenesis and regenerative processes of the respiratory system in non-specific lung diseases and the department of pregnancy pathology of the Far Eastern Scientific Center of Physiology and Pathology of Respiration SB RAS (FESPPR) in line with the requirements of the World Medical Association Declaration of Helsinki "Ethical Principles for Medical Research Involving Human Subjects" (2008). The study was approved by the Far Eastern Scientific Center of Physiology and Pathology of Respiration Ethics Committee. Written informed consent was obtained from all participants.

Statistical analysis was performed using a statistical software package, Statistica 6.0. The mean (M) and standard deviation (SD) were calculated. Analysis of the distribution of values obtained was performed using the KolmogorovSmirnov test. For data with normal distribution, inter-group comparisons were performed using Student's t-test. The Mann-Whitney (U Test) was used to compare the differences between the two independent groups (for nonparametric data). The statistical significance of differences between distributions was estimated by Fischer's t-test for angular transformed proportions. A probability value of $P<0.05$ was considered statistically significant.

\section{Results}

In the study group, the total frequency of all complications of the pregnancy was $180 \%$, which was 4.3 times higher than in the control group. In all pregnant women of Subgroup 1, pregnancy was interrupted due to spontaneous miscarriage. In subgroup 2a, threatened abortion was diagnosed in $60 \%$ of pregnant women; it was significantly greater than in Subgroups $2 \mathrm{~b}(P<0.01)$ and $2 \mathrm{c}(P<0.001)$. In the subgroup $2 \mathrm{~b}$, threatened abortion was observed in $68 \%$, which was significantly greater than in Subgroups $2 \mathrm{a}(P<0.001)$ and $2 \mathrm{c}$ $(P<0.001)$. In Subgroup 2c, a threatened preterm birth was diagnosed in $56 \%$ of pregnant women, i.e., 3.6 times higher than in the control group $(P<0.001)$. Retrochorial hematoma was seen in $24 \%$ of pregnant women in the study group, with a significantly higher frequency in Subgroup 2a compared to Subgroup $2 \mathrm{~b}(P<0.05)$ and Subgroup $2 \mathrm{c}(P<0.01)$. Primary placental insufficiency was diagnosed in $12.7 \%$ of pregnant women in the study group, while it was not found in the control group. In the study group, chronic placental insufficiency, retrochorial hematoma, and chorion previa were diagnosed in $53.3 \%, 24 \%$, and $12.7 \%$ of participants, respectively.

Analysis of the parameters of specific immunity revealed the following features. In the study group, among 
CMV-seropositive women, the frequency of the anti-CMV IgG antibody titer of 1:1600 associated with IgM was $71.3 \%$, while an anti-CMV IgG antibody titer of 1:800 was detected in $28.7 \%$ of cases.

In Subgroup 1, the anti-CMV IgG antibody titer of $1: 1600$ was detected in $80 \%$ of cases, while the anti-CMV IgG antibody titer of 1:800 was detected in 20\% of cases. In subgroups $2 \mathrm{a}, 2 \mathrm{~b}$ and $2 \mathrm{c}$, the anti-CMV IgG antibody titer of $1: 1600$ was detected in $62 \%, 76 \%$ and $78 \%$ of cases, respectively, while the anti-CMV IgG antibody titer of 1:800 was detected in $38 \%, 24 \%$ and $22 \%$ of cases, respectively.

We found a direct relationship between the anti-CMV IgG antibody titer of $1: 1600$ and acute viral respiratory infection during pregnancy $(P<0.01)$, clinical manifestations of which were diagnosed in $74 \%$ of cases. An asymptomatic course of CMVI was observed in $26 \%$ of cases.

Based on these data, we can conclude that a high level of anti-CMV IgG antibody titer may be a diagnostic sign of the intensity of the infection process, and the absence of a reduction in the level of antibody titer is a sign of persisting viremia during pregnancy.

Analysis of the parameters of non-specific immunity also revealed the following features (Table 1). Serum IgA level was significantly less in Subgroup 1 compared to the control group and Subgroups 2a, 2b, and 2c. In addition, the level of total IgG was significantly higher in Subgroup 1 than in the control group, and the level of total IgM was significantly higher than in the control group and Subgroup 2a.

Table 1.

Parameters of humoral immunity in pregnant women with CMVI

\begin{tabular}{|l|c|c|c|c|}
\hline \multirow{2}{*}{ Serum Ig } & \multicolumn{4}{|c|}{ The study group } \\
\cline { 2 - 5 } & Subgroup 1 & Subgroup 2a & Subgroup 2b & Subgroup 2c \\
\hline IgA & $\underline{0.94 \pm 0.06}$ & $\underline{1.05 \pm 0.05}$ & $\underline{1.12 \pm 0.07}$ & $\underline{1.25 \pm 0.06}$ \\
& $2.40 \pm 0.11$ & $2.40 \pm 0.10$ & $2.30 \pm 0.08$ & $2.20 \pm 0.09$ \\
& $P<0.001$, & $P<0.001$ & $P<0.001$, & $P<0.001$ \\
& $P 1, P 2, P 3<0.01$ & $P 4, P 5<0.01$ & $P 6<0.01$ & \\
\hline IgG & $14.0 \pm 0.41$ & $18.00 \pm 0.25$ & $\underline{16.70 \pm 0.74}$ & $\underline{20.0 \pm 0.52}$ \\
& $10.1 \pm 0.09$ & $12.40 \pm 0.11$ & $13.60 \pm 0.11$ & $14.1 \pm 0.12$ \\
& $P, P 1, P 2$, & $P<0.001$, & $P, P 6<0.001$, & $P<0.001$ \\
& $P 3<0.001$ & $P 5<0.01$ & $P 4<0.01$ & \\
\hline IgM & $2.70 \pm 0.07$ & $2.30 \pm 0.05$ & $2.80 \pm 0.09$ & $\underline{2.40 \pm 0.04}$ \\
& $1.22 \pm 0.06$ & $1.20 \pm 0.05$ & $1.12 \pm 0.07$ & $1.09 \pm 0.03$ \\
& $P, P 1<0.001$ & $P<0.001$ & $P<0.001$ & $P<0.001$ \\
& $P 3<0.01$ & & $P 2, P 4, P 6<0.01$ & $P 5<0.01$ \\
\hline
\end{tabular}

Numerator - values of the indexes in the study group; denominator - values of the indexes in the control group. P-between Subgroups $(1,2 a, 2 b, 2 c)$ and the control group; $P 1$ - between Subgroup 1 and Subgroup 2a; P2- between Subgroup 1 and Subgroup 2b; P3 between Subgroup 1 and Subgroup 2c; P4 - between Subgroup $2 a$ and Subgroup 2b; P5 - between Subgroup $2 a$ and Subgroup 2c; P6 - between Subgroup $2 b$ and Subgroup $2 c$.

In Subgroup 2a, serum IgA level was significantly less compared to the control group and Subgroups $2 \mathrm{~b}$ and $2 \mathrm{c}$, but higher than in Subgroup 1. Serum level of total IgM in CMVseropositive women was 1.9 times higher in Subgroup 2a than in the control group, but slightly less compared to Subgroup 1, Subgroups $2 \mathrm{~b}$ and $2 \mathrm{c}$.
In subgroup $2 \mathrm{~b}$, the serum IgA level was less compared to the control group (2 times) and Subgroup 2c. The serum level of total IgM was 2.5 times greater in Subgroup $2 b$ than in the control group and was also higher compared to Subgroups $2 \mathrm{a}$ and $2 \mathrm{c}$. The serum level of total $\mathrm{IgG}$ was also higher in Subgroup $2 \mathrm{~b}$ than in the control group and Subgroup 1, but less than in Subgroups 2a and 2c.

In Subgroup 2c among CMV-seropositive women, the serum IgA level was 1.8 times less compared to the control group, but higher compared to Subgroups 1,2a, and 2b. The serum level of total IgM was 2.2 times greater in Subgroup 2c than in the control group, but less compared to Subgroups $2 \mathrm{a}$ and $2 \mathrm{c}$. The serum level of total IgG was higher in Subgroup $2 \mathrm{c}$ than in all Subgroups.

\section{Discussion}

As we know, CMV persisting in lymphoid organs reveals an activity under the influence of unfavorable factors and conditions that disturb homeostasis [12]. Pregnancy is a powerful factor causing neuroendocrine and immune changes $[13,14]$. An increase in production of anti-CMV antibodies and non-specific antibodies (IgA, IgG, IgM) by the activated $B$ cells is a manifestation of the immune response during CMVI exacerbation in pregnant women [15].

IgM is the earliest factor of phylogenetic and ontogenetic development. IgM production provides the first line of defense in the immune response. Therefore, the detected increase in serum level of total $\mathrm{IgM}$ in all subgroups of the CMV-seropositive pregnant women is a sign of an early inflammatory response during CMVI relapse.

$\mathrm{IgG}$ forms the basic line of specific immunological mechanisms of antiviral defense. The ambiguity of the results regarding the content of total $\mathrm{IgG}$ in the blood of CMVseropositive pregnant women, namely low levels in Subgroups 1 and $2 \mathrm{~b}$ compared to Subgroups $2 \mathrm{a}$ and $2 \mathrm{c}$ in conjunction with a high level of total $\operatorname{IgM}$, indicates a disturbance in immunoreactivity of the B cells. It should be noted that IgM and $\mathrm{IgG}$ are directly involved in the formation of circulating immune complexes (CICs), while increased CIC levels are associated with damaging tissue and vessel walls [16]. In the case of an inadequate response of the immune system, CICs circulate a long time in the blood and are deposited in various organs and tissues. The filter membrane and areas with strong turbulent flow of blood are the most likely sites for CIC fixation. These biological filters are the placentas and kidneys of pregnant women [17,18]. CIC accumulation in the blood is accompanied by the development of rheological disorders and leads to the formation of retrochorial hematomas and the blockage of uteroplacental and fetoplacental circulation. Thromboembolic damage to the placenta and trophoblast caused by dysfunction of vascular endothelium and blood platelets can result in the death of the embryo (fetus). In our early studies, we revealed disturbances in the gestational trophoblast transformation in chorionic villi during CMVI [19], as well as a delay in mesenchyma differentiation towards the formation of vascular endothelium (the reduced villus vascularization) and the degenerative changes in the chorionic 
structures (fibrosis, necrosis of the villous stroma).

According to other authors, a clinically significant placental insufficiency is associated with the presence of fixed immune complexes in the basal membrane of chorionic villi, the syncytiotrophoblast, the basal membrane of endothelium of villus vessels, and the chorionic plate. These abnormalities are accompanied by focal destruction of the marked structures, a massive fibrinoid deposition, and lymphocytic infiltration in the area of damage with the development of circulatory disorders and necrotic processes in surrounding tissues $[17,18]$.

In determining the main immunoglobulins in the blood of CMV-seropositive pregnant women, we revealed a significant reduction in IgA level in association with gestational age. It should be noted that the main function of IgA is neutralization of toxins, an activation of phagocytosis and the complement system, and formation of the antiviral defense for mother and fetus.

In conclusion, the obtained data of the imbalance in the primary and secondary immune response in CMVseropositive pregnant women during relapsed CMVI indicate disturbances in the systemic and local intercellular interactions of immunocompetent cells, which lead to an imbalance in the production of antibodies involved in the elimination of viral agents and to the development of a systemic inflammatory response that complicates the course of pregnancy. CMVI relapse at 7 to 8 weeks of gestation is associated with reproductive losses; a risk for threatened miscarriage, threatened premature labor, and retrochorial hematoma increases significantly with CMVI relapse in the more remote gestational age.

\section{Competing interests}

The authors declare that they have no competing interests.

\section{References}

1. Kulakov VI, Gurtovoiy BL, Ordzhonikidze NV. Cytomegalovirus infection in obstetrics. M.: GEOTARMedia, 2001. [in Russian].

2. Orekhov KV, MV Golubeva, Barycheva LY. Congenital cytomegalovirus infection. Detskie Infektsii 2004; 1:49-55. [in Russian].

3. Alekseeva ML, Ekimov AN, Ekimova VE, Ivanec Yu, Kolod'ko VG. The approaches to the diagnosis of cytomegalovirus infection in obstetrics and neonatology. Problemy Reproduktsii. 2010; 5: 52-56. [in Russian].

4. Vladimirova NYu, Nikitin VG, Chizhova GV. Planning for pregnancy in women with asymptomatic CMV infection. Dal'ne-Vostochniy Zhurnal Infect Pathol. 2012; 21:71-73. [in Russian].
5. Grigorieva EA, Moscow IA, Atyasheva LN, Nikiforova NN. Perinatal cytomegalovirus infection (clinical and laboratory parallels). Epidemiologia Infect Boleznei. 2003;1:40-42. [in Russian].

6. Ozhegov AM, Maltsev SV, Myakishev LS. Clinical and immunological characteristics of active CMV infection with co-infection in children of the first year of life. Pediatriia. 2001; 2:26-31. [in Russian].

7. Asrankulova D, Rizopulu AP, Kurbanov DD. The immune status and anti-inflammatory cytokines in pregnant women with acute cytomegalovirus infection. Zh Microbiol Epidemiol Immunol. 2004;4:84-86. [in Russian].

8. Revello MG, Gerna G. Diagnosis and management of human cytomegalovirus infection in the mother, fetus and newborn infant. Clin Microbiol Rev. 2002; 15(4): 680-715.

9. Dolgikh TI, Dalmatov VV, Gashina EA, Stasenko VL, Kosykh NI, Kmito NL, Pakhalkova EV. Epidemiological aspects of cytomegalovirus infection in babies aged under one. Zh Mikrobiol Epidemiol Immunobiol. 2004;(5):25-30. [in Russian].

10. Kisteneva LB. Cytomegalovirus infection as a problem of perinatal pathology: classification, clinical presentation, differential diagnosis, treatment, and prevention. Ross Vest Perinatol Pediatrii. 2003; 5:52-56. [in Russian].

11. Sidelnikova VM, Dadalyan DG, Van'ko LV, Sukhih GT. Cytomegalovirus infection in women with recurrent miscarriage. Akush Ginekol (Mosk).1996;4:21-24. [in Russian].

12. Klimov VA. Infectious diseases and pregnancy. M.: MEDpress; 2009. [in Russian].

13. Andrievskaya IA. Morphofunctional characteristics of the placenta and disorders in metabolism of hormones and bioactive substances in pregnant women with herpes virus infection. Abstract of PhD thesis. Irkutsk, 2004. [in Russian]. 14. Lucenko MT, Solovieva AS, Andrievskaya IA. Mechanisms of changes in immune system of pregnant women with herpes viral infection. Novosibirsk- Blagoveshchensk: Publishing House of ASMA; 2007. [in Russian]. 15. Moskalev AV, Sboychakov VB. Infectious Immunology. In: Lobzina YuV, editor. SPb.: Foliant; 2006. [in Russian]. 16. Konstantinova NA. Immune complexes and tissue damage. M.: Medicine; 1996. [in Russian].

17. Gabelova KA, Arutyunyan AV, Zubzhitskaya LI, et al. Fixed immune complexes and NO-synthase activity in the placenta during preeclampsia. Vestnik Ross Assotsiatsii Akush Ginekol. 2000; 1:22-24. [in Russian].

18. Starostina TA, Belokrinitskiı̌ DV, Kudriashova NM, Razmakhnina NI, Goloshchapova OV, Seredina TA. Circulating immune complexes and immunoglobulins A, M and $\mathrm{G}$ in the mother-fetus system in normal pregnancy and gestosis. Akush Ginekol (Mosk). 1992; 1: 19-22. [in Russian]. 19. Babenko OP. Morphofunctional characteristics of the placenta during exacerbation of cytomegalovirus infection in the early stages of gestation. Bull Physiol Pathol Resp (SB RAMS) 2013; 47:72-76. [in Russian]. 